\title{
FUNDAMENTOS Y CLAVES PARA EL FUTURO DE LOS SERVICIOS SOCIALES Y EL TRABAJO SOCIAL
}

\author{
THE BASES AND KEYS FOR THE FUTURE OF THE SOCIAL WORK AND \\ SERVICES
}

Antonio Gutiérrez Resa Universidad Nacional de Educación a Distancia, Madrid. España/Spain agutierrez@poli.uned.es

Recibido/Received: 04/03/09

Modificado/Modified: 25/09/09

Aceptado/Accepted: 06/10/09

\section{RESUMEN}

Los valores actualmente emergentes de los Servicios Sociales y el Trabajo Social tratan de compatibilizar el ámbito de los servicios públicos con aquellos que oferta el mercado. Ahora bien, sin la garantía del Estado que vele por la universalidad y por la proximidad a lo local, así como por la calidad de los Servicios Sociales y el Trabajo Social, es el mercado quien tratará de resolver la gestión de las necesidades humanas. En adelante, los fundamentos y claves de los Servicios Sociales y el Trabajo Social han de requerir, al menos, de la escucha y participación de los usuarios, así como de los contenidos relacionales.

\section{PALABRAS CLAVE}

Servicios públicos, mercado, escucha, participación, contenidos relacionales.

\section{ABSTRACT}

The actual values emerging from the Social Services and the Social Work treat to make compatible the Public Services Field with those which the Market offers. However, without the State Guarantee which to look after universality and local proximity, so after the quality of the Social Services and Social Work, it is the Market which treat to resolve the management of the human being. In the future the bases and keys of the Social Services and Social Work have to require, at least, from the Beneficiaries listening and participation, the same way as relation contents.

\section{KEYWORDS}

Public Services, Market, Listening, Participation, Relation Contents.

\section{LOS NUEVOS VALORES DE LOS SERVICIOS SOCIALES Y EL TRABAJO SOCIAL}

En este apartado entresacamos aquellos valores que han servido para cambiar y para reformar los Servicios Sociales Comunitarios desde sus inicios. También subrayaremos los valores que han permanecido $\mathrm{y}$, desde luego, apuntaremos a aquellos valores que 
consideramos emergentes y que entendemos que deben propiciarse de cara al futuro de los Servicios Sociales Comunitarios y el Trabajo Social en España. De esta manera consideramos estar dando respuesta a los fundamentos para el futuro de los Servicios Sociales y el Trabajo Social ${ }^{1}$.

Hemos de recordar que con el Plan CCB se propiciaba un conjunto de valores sustentadores así como prestaciones y equipamientos para el desarrollo de una comunidad cristiana de bienes y destinados a las clases populares más desfavorecidas. A partir de entonces, los llamados Servicios Sociales Comunitarios han ido cambiando al mismo tiempo que han conservado ciertos valores y, desde luego han hecho emerger una serie de tendencias-valores que apuntan a un futuro de los citados Servicios algo distinto de lo que fueron y de lo que son en la actualidad en España.

El cuadro $\mathrm{N}^{\circ} 1$ puede ayudarnos a clarificar lo que estamos diciendo. Comprobamos que los valores permanentes como irrenunciables reúnen valores finales e instrumentales.

Cuadro $\mathrm{N}^{\mathrm{o}} 1$. Sobre valores de cambio, valores permanentes y valores emergentes para el futuro de los Servicios Sociales Comunitarios

\begin{tabular}{|l|l|l|}
\hline Valores de Cambio & Valores Permanentes & Valores Emergentes \\
\hline Flexibilidad & Universalidad & Público/mercado \\
\hline Un derecho & Calidad & Competitividad \\
\hline & Cooperación/Solidaridad & \\
\hline $\begin{array}{l}\text { Formación permanente } \\
\text { Participación/Coordinación }\end{array}$ & Proximidad/Seguimiento & $\begin{array}{l}\text { Precisión/Rapidez } \\
\text { Especialización } \\
\text { Complejidad de equipos }\end{array}$ \\
\hline
\end{tabular}

Fuente: Elaboración Propia a partir del Plan Concertado y Servicios Sociales. Modelos y Perspectivas. Una aproximación internacional (Gutiérrez Resa, A. y Servós Marcuello, Ch. 2008: 191)

Los valores de cambio han sido capaces de transformar el sistema de prestaciones y equipamientos y los valores emergentes nos indican que debemos estar atentos a las nuevas tendencias que se muestran con fuerza para elevar el bienestar de los ciudadanosusuarios de los Servicios Sociales Comunitarios.

Los nuevos valores de cambio como la formación permanente y la participación/coordinación, son valores instrumentales que según los trabajadores sociales se consideran imprescindibles para adecuarse a los tiempos actuales y futuros. Los citados profesionales requieren de formación continuada para entender y atender los nuevos problemas sociales (inmigración y autonomía-discapacidad, entre otros). La participación la indican los usuarios que desean algo más que recibir prestaciones, porque prefieren ver unidas la gestión técnica y la humanización de los servicios. La coordinación entre las tres administraciones, así como dentro de las autonomías, es un clamor de usuarios y profesionales para avanzar en la eficacia y en la eficiencia, aproximando protocolos de diversos servicios públicos que, en definitiva lleguen a prestar Servicios Sociales de calidad.

Entre los valores permanentes subrayamos ahora el seguimiento, que es una de las características de la proximidad. Seguimiento de los casos, de los problemas que padecen los usuarios. Se trata de estar en contacto, sobre todo físico, con los usuarios para detallar el proceso a seguir, realizar los informes y tener un conocimiento fiel de los problemas que existen y de cómo plantear soluciones adaptadas a las situaciones de los usuarios. Seguramente es uno de los valores finalistas que deben cuidarse en la actualidad más que nunca para poder captar los cambios sociales que tienen lugar en la sociedad española ${ }^{2}$. 
Consideramos que la gestión se deshumaniza y los usuarios reclaman poder hablarconversar con los trabajadores sociales, con los auxiliares de hogar o con las operadoras de la teleasistencia. Lo hemos comentado con anterioridad y lo volvemos a expresar de otro modo: los usuarios piden eficacia, piden rapidez y precisión, pero también piden atención estrictamente humana. No ser tratados como en una agencia de viajes, sino verse y sentirse acompañados. Es lo que denuncian usuarios de Servicios Sociales del norte de Europa como Dinamarca, o bien participantes de instituciones religiosas centro europeas como Alemania y por añadidura voluntarios de entidades sin ánimo de lucro en España ${ }^{3}$.

Entre los valores emergentes, además de los ya conocidos y que aparecen en el cuadro $\mathrm{N}^{0} 1$ figura la precisión, la rapidez y la especialización y complejidad de los equipos profesionales. Son valores instrumentales y han surgido por el crecimiento constante de usuarios que se atienden diariamente en los Servicios Sociales Comunitarios. Sin embargo se exige rapidez, porque los usuarios demandan prontitud y menos listas de espera en prestaciones como el Servicio de Ayuda a Domicilio, ayudas económicas y ayudasprestaciones para la discapacidad.

\section{LOCALISMO Y UNIVERSALIDAD DE CRITERIOS EN LOS SERVICIOS SOCIALES Y EL TRABAJO SOCIAL}

Se dice con frecuencia que los Servicios Sociales han de estar próximos a los usuarios. Quiere decirse que han de resolverse los problemas concretos de la gente que vive en una determinada localidad. Sin embargo los criterios, los principios, han de ser universales, aunque tengan que hacer frente a las demandas locales de los usuarios. Lo que decimos ha de entenderse en un contexto nacional-europeo y global, que, dicho sea de paso plantea problemas de ejecución. ¿Qué es lo que sucede habitualmente? Que todos deseamos experimentar que el bienestar nos alcance no aceptando sin más cualquier solución de apariencia o alcance universal distante de nuestra situación y contexto. Vivamos en el inmortal pueblo donde nació Goya, Fuendetodos, o en Madrid o Barcelona, cualquier ciudadano que requiera de un informe social, que necesite el servicio de ayuda a domicilio, SAD, o que le orienten cuando se trata de cuidar a un familiar que pierde autonomía y requiere de un plan detallado de apoyo, desea ser atendido y que el problema se solucione. No le servirá a ningún ciudadano escuchar que los Servicios Sociales alcanzan una cobertura máxima en toda España o que son prestaciones universales o para todos sin ver zanjado su problema. ¿Por qué, entonces, este afán de hacer creer que lo que aparece en las imágenes o se lee en la prensa, constituye un disfrute de todos los ciudadanos? Porque existe una auténtica lucha por mostrar como universal lo que constituye un contenido específico que acaba ocupando o usurpando esa universalidad y sosteniendo su ineficacia. "Así, en el rechazo del Estado Social reiterado por la Nueva Derecha estadounidense, la idea de la ineficacia del actual Welfare system ha acabado construyéndose sobre, y dependiendo del, ejemplo puntual de la joven madre afroamericana: el Estado Social no sería sino un programa para jóvenes madres negras. La "madre soltera negra" se convierte, implícitamente, en el reflejo "típico" de la noción universal del Estado Social... y de su ineficiencia".

En términos generales, hablamos de universalidad y de localismo para referirnos a la necesidad de compatibilizar lo que es propio del ámbito concreto y sus problemas así como a la defensa de principios universales y, claro está, aplicables a todos los ciudadanos. 
Sin embargo, tenemos la impresión de que las cosas no caminan en la dirección que decimos: cualquier atención concreta, local, que se realice en materia de Servicios Sociales, se indica que es expresión de universalidad. Y no es así; lo que se hace y propaga más bien tiene que ver con una adaptación a lo que toca, sean inmigrantes o personas con discapacidad, sin aumentar apenas los presupuestos, las plantillas y las mismas prestaciones. Luego la universalidad la ejemplifican con lo que atienden localmente, aunque sea sectorial y con escasos medios materiales y profesionales. El ámbito local queda desubicado, desconectado, por mucho que lo exhiban o manifiesten como representante universal. La llamada proximidad en los Servicios Sociales comunitarios se está convirtiendo entonces en una quimera.

La proximidad a lo local, al menos teóricamente, es lo que parece que orienta mejor la acción de los Servicios Sociales. Habremos de comprobar en qué medida lo más próximo participa de esa universalidad de principios que parece encerrar cierta naturaleza que poseen todos los seres humanos. Es lo que indican Habermas y Apel. Por el contrario el filósofo norteamericano Rorty es menos ambicioso al indicarnos que "No se esperará que se cree una única comunidad de justificación por la habilidad para comunicarse, puesto que la habilidad para usar el lenguaje es, como el pulgar prensil, una astucia más que los organismos han desarrollado para aumentar sus probabilidades de supervivencia"5. En consecuencia, hemos de estar atentos para desentrañar aquellas estrategias que hacen posible resolver los problemas locales, al mismo tiempo que estamos dispuestos a compartir con otros nuestras soluciones, ampliando de este modo el pensamiento y la acción locales.

Las diversas maneras de atender las necesidades a través de los Servicios Sociales como lo es por medio de la concertación, las fundaciones y el mercado, entre otras fórmulas, traiciona la universalidad defendida por los Servicios Sociales públicos. Lo mismo está sucediendo con hospitales y hasta con cárceles. Luego se desvanece o deteriora el Estado-Nación que venía siendo el garante de la calidad y universalidad de las prestaciones en el Estado de Bienestar.

Sin la garantía de un Estado que vele por la universalidad y calidad de los Servicios Sociales y el Trabajo Social, es el mercado y las multinacionales quienes resolverán la gestión de las necesidades humanas. Los propios medios de comunicación nos indican cómo vestirnos mejor, cómo educar a un niño, cómo alimentarnos, cómo relajarse y divertirse. Ante la dificultad de poder decidir con toda la información necesaria, es el mercado quien te oferta soluciones. Entendemos que por el temor a decidir mal, la información y orientación están siendo, cada vez más, objetivos prioritarios del Trabajo Social y los Servicios Sociales en una sociedad que vive el riesgo, el miedo ante decisiones que deben tomarse. Luego las soluciones de mercado no existen porque él mismo alimenta esa profunda confusión en donde todo es posible: acabar con la constancia del esfuerzo o progresar por la especulación ${ }^{6}$. Disponer entonces de profesionales del Trabajo Social en los Servicios Sociales que son para todos, es fundamental para atender a los usuarios, explicarles cómo funcionan las cosas, calmarlos y asesorarles en las cuestiones básicas y complementarias de la vida. Orientarlos en la realización del yo que abandonó la comunidad y "ha entrado en una serie de asociaciones móviles, cambiantes, irrevocables, a menudo meramente destinadas para fines sumamente específicos"7. Los usuarios, anclados algunos, en modos tradicionales de interpretar y resolver los problemas, no tienen tiempo y dinero para consultar comités de ética, científicos y autoridades locales o supralocales. Les quedan, entre otros profesionales, los trabajadores sociales que tienen 
experiencia en los problemas humanos y en el modo de gestionarlos y resolverlos o encauzarlos, con acompañamiento y trato humano. Los profesionales del Trabajo Social pueden llegar, por tanto, a ligar, a concordar, la mayor seguridad que desean los ciudadanos con las mayores dosis de libertad. La no concordancia entre más seguridad y más libertad es lo que constituye, precisamente, la llamada sociedad del riesgo ${ }^{8}$.

Se trata de trasmitir a los usuarios, desde el Trabajo Social y Los Servicios Sociales que la responsabilidad de las decisiones que toman las deben asumir ellos mismos, a la vez que el análisis y seguimiento de los problemas cuenta con la capacidad y el compromiso profesional de los trabajadores sociales. No se trata entonces ni de ser jueces ni de sustituir las responsabilidades paternas. Más bien conviene recuperar el trato humano con los usuarios, escucharles atentamente, conocer en detalle e in situ los pormenores que configuran tanto los problemas como el contexto social más próximo donde tienen lugar.

Finalizaremos este apartado haciendo referencia a la atención que prestan los Servicios Sociales en un pueblo, en el ámbito local. Se trata de la localidad de Fuendetodos. El pueblo natal de Goya queda encuadrado en la atención comarcal del Campo de Belchite junto a otros catorce pueblos con 5.674 habitantes en total. Hay dos trabajadoras sociales para la comarca y cuando una de ellas disfruta de vacaciones (15 días) no existe sustitución de la trabajadora social haciéndose responsable de los Servicios Sociales comunitarios la otra profesional. Ahora bien, en este último caso la trabajadora social no se desplaza a los pueblos y atiende desde Belchite. También cuentan en la citada comarca con un auxiliar administrativo y ocasionalmente (cuando lo necesitan), con un abogado y un psicólogo.

La trabajadora social visita Fuendetodos cada 15 días los lunes y durante dos horas. Lo que más demandan en la comarca es la Ayuda a Domicilio, la teleasistencia y el transporte social. El transporte social, que es una novedad en la comarca, es adaptado, lleva a los usuarios a revisiones médicas, para hacerse análisis, etc. cuando el usuario no dispone de medios o requiere de un transporte con determinadas condiciones, según los casos.

Lo que más valoran los usuarios es que les escuchen, que les digan la verdad y que no les creen falsas expectativas. Sin embargo, existen problemas como la soledad que padecen bastantes personas mayores y cuyas soluciones pueden paliar superficialmente el problema señalado. Los dos profesionales de la comarca Campo de Belchite indican la necesidad de desarrollar la prevención. Prevención en materia de conflictos familiares, violencia, consumo de drogas, fracaso escolar, y tantos otros problemas que requieren de la actuación de información y asesoramiento de los profesionales del trabajo social en los 15 pueblos de la comarca. Podemos añadir que el nivel de cumplimiento de la Ley de Dependencia alcanza el $85 \%$ de modo que la mayoría de las personas que tramitaron sus solicitudes reciben ya sus prestaciones, incluidos los atrasos en las percepciones económicas.

En algunos pueblos de la comarca, que apenas si tienen 13 vecinos, llegan a interpelar amablemente a la trabajadora social indicándole que no les visite tantas veces, que no es necesaria su asidua presencia. Hemos de tener en cuenta que se trata de 13 vecinos y que de no existir una necesidad puntual, prefieren solucionar las cosas entre ellos, sin la presencia de alguien de fuera del pueblo. En consecuencia, es importante la proximidad de la que hablamos y valoramos en los Servicios Sociales comunitarios; es imprescindible que seamos capaces de matizar, para saber algo más de aquellos valores 
que hemos venido considerando como algo tradicional y que en el caso que mencionamos se presenta como una excepción o con matices.

\section{GESTIÓN EFICAZ Y PASIVIDAD DE LOS USUARIOS}

"Las estructuras simplemente no dan lugar a la participación. Ni para ninguna interacción social más extensa. Asta no es muy sociable y nunca ha pedido ayuda a nadie, está agradecida por la ayuda que recibe pero siente pena por los trabajadores sociales y sus condiciones de trabajo. La modernización ha sido excesiva"9. Hablamos de una mujer, Asta, que es una danesa de 84 años, divorciada, con tres hijos, aunque dos de ellos viven fuera del país y el tercero a más de 300 millas. Aunque los vecinos le suelen ayudar, Asta que apenas ve a su familia, tiene un círculo social muy limitado.

Como tantas personas mayores en España, Asta en Dinamarca recibe una ayuda de atención para personas mayores. Por las mañanas una trabajadora social (en España una auxiliar de hogar del Servicio de Ayuda a Domicilio, SAD) le ayuda a levantarse, vestirse y le prepara el desayuno y la comida. Por la noche una trabajadora le sirve la cena y otra, del turno de noche, le ayuda con la higiene personal. En España, lo habitual tratándose de una persona con más de 80 años es que la cuide la familia o que reciba el SAD dos días y dos horas cada día para limpiar, ordenar la casa y poco más, en el mejor de los casos. Sin embargo, hemos de añadir que el contexto está evolucionando rápidamente de manera que es necesario cuidar a los cuidadores, mientras la propia familia pierde efectivos, si consideramos las posibilidades reales de ayudar-cuidar a sus mayores.

Hortensia Paniagua que vive en Barrado, un pueblo cacereño de unos 500 habitantes en el Valle de los Cerezos, tiene 62 años y su jornada comienza cuando tiene que levantar a su suegra, la abuela de 91 años, hacerle el desayuno, ayudarle con la higiene personal y ponerle en la mano las pastillas que le tocan. Cuando no está su suegra está su madre, otra anciana de 82 años con parkinson y tensión alta. A Hortensia le quedan sólo dos meses libres al año para dedicarlos a sí misma, a su marido y a sus nietos, que ya tiene tres. Así lleva años. Casi no se acuerda de lo es ir a dar una vuelta sin preocupaciones rondándole la cabeza: "Salgo un rato los domingos pero estoy venga a mirar el reloj, no estoy tranquila, no puede ser" ${ }^{\text {. }}$. Por eso mismo existe el servicio de respiro para cuidadores pero que alcanza a muy pocas personas, a pesar del elevado número de familias que se sienten desbordadas por los cuidados que deben prestar a sus pequeños y personas dependientes.

En Dinamarca, Asta nos dice por la experiencia adquirida que los trabajadores sociales soportan hoy una carga de trabajo mucho más pesada que antes. Entre las estrategias de modernización, la efectividad y la calidad de las mejoras han sido la meta del trabajo de desarrollo. "Los trabajadores sociales no tienen tiempo para hablar ni para tomar café". Y aunque las tecnologías aumentan la efectividad del sistema, Asta piensa que la precisión de los horarios, los ordenadores de mano y los sistemas de definición de asistencia son exagerados y poco fructíferos a la hora de asistir. En otras palabras, ya no queda tiempo para vaguear o beber café; como cuando los trabajadores sociales eran casi como una plantilla de esclavos. Así era hace 40 años en Dinamarca y entonces los jefes no distribuían el tiempo sino que los mismos trabajadores sociales decidían junto con el usuario qué era mejor para ellos. Si antes era mejor o peor que ahora, lo cierto es que hace 40 cuarenta años los trabajadores sociales y los usuarios disponían de un espacio para discutir entre ellos sobre la asistencia. "Asta piensa que el mayor problema es el de la definición tan restringida de lo que 
es asistencia. Se decide con antelación qué servicios va a recibir. Asta siente que está en una situación de tutela absoluta. Nada queda sin responder en sus cuidados. La situación no es muy prometedora si la intención es de ayudar. Se espera que ella participe en la ayuda y que actúe responsablemente" ${ }^{, 11}$. Asta por tanto, responde que sólo actúa como las estructuras le dictan, es decir de manera pasiva y aliviada.

Efectividad, calidad, participación, son términos, valores que han aparecido en lo desarrollado en este apartado. Podríamos añadir otros valores que son mencionados por usuarios daneses y españoles que tienen experiencia por la asistencia que reciben. Nos vamos a referir a la flexibilidad de la asistencia diaria. La determinación personal de los usuarios si ha de combinarse ha de ser con la flexibilidad y no precisamente con servicios de horario apretado. No obstante, se han de tener en cuenta las circunstancias y normas que han de cumplir los profesionales que prestan los servicios requeridos y que limitan necesariamente la libertad de elección de los usuarios. Aún así lo ideal sería que el usuarioconsumidor supiera qué era bueno para él o ella y que a imitación de la teoría de mercado, tuviera su propio subsistema en los servicios de asistencia del sector correspondiente.

La clásica asociación profesional-usuario se tambalea. En consecuencia surgen nuevas formas de organización de la asistencia social que intentan crear servicios en los que aquellos que necesitan ayuda puedan participar activamente prestando ayuda, y actuando en los niveles tanto de la planificación como de la prestación de la ayuda. Al mismo tiempo, sin embargo, se ponen en práctica nuevas formas de control y se introducen nuevos medios o instrumentos electrónicos.

Lo que parece ir quedando claro, según la experiencia adquirida con los usuarios de los Servicios Sociales a través de los trabajadores sociales es, que las personas que reciben las prestaciones se sienten solas porque no participan. Sin embargo, el desarrollo y progreso de los Servicios Sociales y el trabajo social que apunta a una gestión eficaz y de calidad de los mismos, interpreta que ha de suponer precisión informática de las funciones y los tiempos a emplear. De este modo se gestionan las necesidades como elementos de dominio y control sin que quepan ingredientes como la conversación, el entretenimiento, y el interés humano por el usuario por parte de los profesionales. Es curioso que en buena parte de los países europeos se haya difundido el modelo del care management que ha provocado modificaciones sustanciales en el rol del trabajador social. Se garantiza un cierto nivel de bienestar a precios reducidos. Y entre tanto se agranda la separación que hemos visto en Dinamarca, entre quien presta el servicio y quien lo recibe. Se asiste a un desarrollo de intervenciones diferenciadas en la comunidad así como a la aparición de una concesión en las respuestas a los deseos en términos de paquetes personalizados de servicios $^{12}$.

La mayor presencia del mercado hace posible que ni las instituciones ni la comunidad asuman responsabilidades, siendo el propio sujeto quien deba elaborar el diseño de cómo satisfacer sus necesidades. ¿Y qué es lo que ocurre?. Lo que sucede es que los sujetos, "los individuos, atrapados como siguen en el paradigma modernista tradicional, buscan desesperadamente una instancia que legítimamente pueda ocupar la posición del "Sujeto que sabe" y venga a avalar sus decisiones...".". En la práctica, se genera la apariencia de elegir libremente entre las ofertas del mercado cuando apenas si existen profesionales que les puedan asesorar y hacer cierto seguimiento.

Puede estar sucediendo que si los usuarios de los Servicios Sociales tratan con profesionales del Trabajo Social presos de roles y automatismos burocráticos de más control que participación, reaccionen con el doble lenguaje. Todo se tramita, todo se 
gestiona, de tal manera que el usuario reduce al trabajador social a un objeto al que trata de convencer o de seducir, ocultando sus verdaderas necesidades. Habla sin cesar ocultando sus necesidades para no sufrir desengaños. Entonces el trabajador social oficia el papel que el mismo usuario le reconoce: de analista superficial de problemas sociales, en cuyo entramado no puede entrar porque supondría reconocer la seriedad de los problemas y su atención inmediata. Si el usuario se ha apercibido de la situación y entiende del papel pasivo y burocrático que ejerce el trabajador social en los Servicios Sociales, entonces, mientras el profesional permanece "en silencio", el usuario actúa, habla para que nada pase, para que no se pronuncie la palabra o palabras que pongan en evidencia la "irrelevancia de su incesante hablar".

¿Qué hacer para salir como profesional de esas situaciones impasibles e inertes? Una de ellas sería una adaptación pasiva y resignada por parte de los profesionales del Trabajo Social. En otras palabras, los ideales de la profesión se sustituyen por un comportamiento cínico y de renuncia o abandono, al tiempo que se refugian pasivamente en una práctica burocrática del trabajo. Una segunda alternativa está consistiendo en claudicar del ejercicio de la profesión en el ámbito público, ejerciendo en el ámbito privado que supuestamente está más acorde con los valores de la profesión. Lo cierto es que gran parte del ámbito privado, sobre todo sin ánimo de lucro, se alimenta o sostiene a través de los convenios y concertaciones y por tanto bajo el dominio y lógica propios de los entes públicos. Una tercera respuesta consiste en realizar una adhesión aparente a las políticas dominantes. Resistencia pasiva que se traduce en desarrollar aparentemente el trabajo según las normas, aunque de hecho se continúe trabajando en consonancia con la profesionalidad. Se cubren las apariencias en el trabajo burocrático administrativo y al mismo tiempo se cuidan las relaciones significativas con los usuarios. La cuarta alternativa o respuesta, consistiría en establecer mayores relaciones entre trabajadores sociales, y los ámbitos del voluntariado y las asociaciones de inmigrantes, asociaciones de vecinos y aquellas más próximas a los problemas sociales que padecen los ciudadanos. De hecho, las asociaciones de inmigrantes en las grandes ciudades, desarrollan funciones de información, mediación y hasta de sustitución del Trabajo Social.

Es necesario apostar por la iniciativa y la creatividad del Trabajo Social y los Servicios Sociales. En este sentido conviene recordar que la interpasividad está relacionada con la actual situación global que se vive en el mundo. "El ámbito de las relaciones capitalistas de mercado constituye la Otra escena de la supuesta repolitización de la sociedad civil defendida por los partidarios de las "políticas identitarias" y de otras formas postmodernas de politización: todo ese discurso sobre esas nuevas formas de la política que surgen por doquier en torno a cuestiones particulares (derechos de los homosexuales, ecología, minorías étnicas...), toda esa incesante actividad de las identidades fluidas, oscilantes, de las múltiples coaliciones ad hoc en continua reelaboración, etc., todo eso tiene algo de profundamente inauténtico y nos remite, en definitiva, al neurótico obsesivo que bien habla sin cesar bien está en permanente actividad, precisamente con el propósito de asegurarse de que algo -lo que importa de verdad-no sea molestado y siga inmutable. El principal problema de la actual post-política, en definitiva, es que es fundamentalmente interpasiva" ${ }^{, 14}$.

\section{SOBRE INVERSIÓN ECONÓMICA Y RESPONSABILIDAD PROFESIONAL}

El gasto en Servicios, incluidas las plantillas de los trabajadores entre los que son protagonistas los trabajadores sociales, es de capital importancia. Igualmente se ha de 
considerar en qué condiciones asumen sus responsabilidades profesionales los trabajadores sociales. Dicho de otro modo, si han de atender a un número elevado de usuarios y con dificultades para hacer seguimiento de casos (elevado número de informes sociales en la actualidad con motivo de la aplicación de la Ley de Dependencia), no se atiende con horario de tarde estandarizado y no hay servicio de urgencia, la responsabilidad profesional es difícil de ejercer. No es fácil dar una respuesta: los responsables políticos han de aumentar las plantillas, establecer horarios de mañana y tarde así como de urgencia, apostando claramente por los Servicios Sociales Comunitarios, entre otras medidas.

En términos de inversión económica en materia de Servicios Sociales comunitarios, son los ayuntamientos los que más aportan. Son los más próximos a los ciudadanos y los que más esfuerzos económicos realizan para atender las necesidades de sus vecinos. En el caso del Plan Concertado del año 2005 pueden verse claramente en el cuadro $\mathrm{N}^{\mathrm{o}} 2$ que son las Corporaciones Locales los que aportan el 60\% de la financiación del Plan Concertado.

Cuadro $\mathrm{N}^{\mathrm{o}}$ 2. Financiación total de Plan Concertado 2005

\begin{tabular}{|l|c|c|}
\hline \multirow{2}{*}{ ADMINISTRACIÓN } & \multicolumn{2}{c|}{ APORTACIONES } \\
\cline { 2 - 3 } & CANTIDAD & $\%$ \\
\hline Ministerio de Trabajo y Asuntos Sociales & $90.802 .350,00$ & 10,92 \\
& & 27,95 \\
\hline Comunidades Autónomas & $232.403 .816,74$ & 60,93 \\
\hline Corporaciones Locales & $506,625.803,54$ & 0,20 \\
\hline Remanentes incorporados & $1.669 .109,41$ & 100,00 \\
\hline Total & $831.501 .079,69$ & \\
\hline
\end{tabular}

Fuente: Plan Concertado 2005-2006

Es la razón por la que los municipios viven directamente los problemas: los vecinos les piden soluciones a ellos porque están más cerca. Y entre las soluciones están aquellas que tienen que ver con los Servicios Sociales que demandan los vecinos. Así las cosas La Federación Española de Municipios y Provincias (FEMP) piden mayor margen para subir los impuestos propios, participación en los estatales y autonómicos y un fondo de nivelación que disminuya las diferencias entre municipios ${ }^{15}$.

La red nacional del Plan Concertado cuenta, básicamente, con Centros de Servicios Sociales, albergues y centros de acogida, que son atendidos por 28.269 trabajadores. Los Centros de Servicios Sociales, 1.258, suponen el 98\% de todos los equipamientos, mientras que hay 13 albergues y 13 centros de acogida. Es fácil suponer que son los Centros de Servicios Sociales quienes atienden a un mayor número de personas: a 4.641.446 usuarios. Atienden en este último caso a personas mayores y a familias con prestaciones tales como la Ayuda a Domicilio ( $75 \%$ del gasto en los Centros de Servicios Sociales) y con la Información y Orientación, respectivamente.

Cuadro No 3. Relación laboral trabajadores/centros del Plan Concertado

\begin{tabular}{|l|c|c|c|c|c|}
\hline $\begin{array}{c}\text { Relación } \\
\text { Laboral }\end{array}$ & $\begin{array}{c}\text { Trabajadores } \\
\text { en CSS }\end{array}$ & $\begin{array}{c}\mathbf{N}^{\mathbf{o}} \\
\text { Trabajadores } \\
\text { en albergues }\end{array}$ & $\begin{array}{c}\mathbf{N}^{\mathbf{0}} \text { Trabajadores } \\
\text { en Centros de } \\
\text { acogida }\end{array}$ & Total & $\%$ \\
\hline Plantilla & 11.525 & 110 & 125 & 11.760 & 41,26 \\
\hline Prestaciones & 16.744 & & & 16.744 & 58,74 \\
\hline TOTAL & 28.269 & 110 & 125 & 28.504 & 100,00 \\
\hline \multicolumn{7}{|c|}{ Memoria. Plan Concertado 2005-2006 }
\end{tabular}


En 2005 un total de 28.504 personas han trabajado en el marco del Plan Concertado. Los contratados con cargo a los programas de los centros han sido $16.744(58,74 \%)$ y en las plantillas de los mismos 11.760 (41,26\%), de los 28.504 trabajadores.

Los trabajadores sociales, que son los profesionales básicos de los centros de Servicios Sociales, son mayoritarios en la composición de las plantillas $(38,64 \%)$, mientras que los trabajadores contratados con cargo a las prestaciones (Apoyo a la Unidad Convivencial y Ayuda a Domicilio), el 78,3\% están compuestos por auxiliares de hogar. Por otra parte, si consideramos a los funcionarios y a las personas que tienen un contrato laboral fijo, un $66 \%$ del personal de plantilla puede afirmarse que son fijos. Claro que, han de anotarse las condiciones de cada uno de los ayuntamientos a la hora de mantener sus correspondientes plantillas en materia de Servicios Sociales. Las diferencias pueden ser notables; lo mismo sucede con la ratio resultante de trabajadores respecto a la población, que es de 1.268 habitantes por trabajador. Sin embargo en Ceuta es de 6.786 habitantes por trabajador, mientras que en Extremadura es de 671 habitantes por trabajador. Los trabajadores de plantilla, tienen en general (88\%) una dedicación completa a partir de 35 horas; sin embargo, entre aquellos que se contratan con cargo a las prestaciones predomina, con el $66 \%$ una dedicación menor.

En la tabla $\mathrm{N}^{\circ} 4$, que figura a continuación, aparecen los costes salariales anuales de trabajadores en plantilla y prestaciones de los Centros del Plan Concertado en el año 2004. A primera vista se ponen de manifiesto las diferencias existentes en toda España, en cualquiera de las tipologías de trabajadores que figuran en el citado cuadro. Baste con observar lo que sucede con los trabajadores sociales en plantilla y prestaciones, teniendo presente la media, el coste máximo y el coste mínimo salarial.

También se pueden apreciar las diferencias salariales en las diversas Comunidades Autónomas españolas. Es más, los cambios de un año para otro referidos a los costes salariales que figuran en el cuadro $\mathrm{N}^{0} 4$ son curiosos. Si en el año 2004 un monitor (Prestaciones) cobraba en Aragón 3.639€, mientras que el máximo suponían 14.239€ en Canarias el mismo año, en el cuadro que presentamos ahora, un año más tarde, es La Rioja quien paga a un monitor $679 €$ en Prestaciones.

Podemos entonces indicar, a juzgar por los datos que presentamos, que la relación que deseamos establecer entre responsabilidad profesional y medios disponibles no es fácil de mantener. Si tenemos en cuenta los datos anteriores, otra de las cuestiones importantes es saber si existe necesidad de ampliar el número de profesionales. En el caso concreto de Andalucía y de los Trabajadores Sociales el 58\% de los mismos indican que son muy necesarios y el 22\% que son necesarios en Andalucía. En tal caso, en torno al $80 \%$ de los profesionales indican como necesaria y más que necesaria la incorporación de Trabajadores Sociales. Y en el caso de Aragón un 63,2\% de los trabajadores sociales indican que no son suficientes en número para atender las demandas que se les plantean ${ }^{16}$.

Los Trabajadores Sociales son los profesionales que desde los inicios del Plan Concertado han dado la identidad a los Servicios Sociales Comunitarios. Así ha sido y lo es en la actualidad porque en base a ellos se genera y desarrolla la mayor parte de las actuaciones que tienen lugar en los centros de Servicios Sociales Comunitarios y Unidades de Trabajo Social. Según ellos, es excesivo el volumen de burocracia/tramitación administrativa exigido, no existe suficiente coordinación entre las administraciones central, autonómica y local al faltar una estrategia conjunta y el exceso de burocracia así como la falta de comunicación. No obstante hay interés de los responsables de los ayuntamientos para con los Servicios Sociales de Base. 
Cuadro $\mathrm{N}^{\mathrm{o}} 4$. Costes salariales anuales de trabajadores en plantilla y prestaciones de los Centros del Plan Concertado

\begin{tabular}{|l|l|l|}
\hline \multirow{2}{*}{} & \multicolumn{2}{|c|}{ Trabajadores } \\
\cline { 2 - 3 } & \multicolumn{1}{|c|}{ Plantilla. } & \multicolumn{1}{|c|}{ Prestaciones. } \\
& Coste Medio.C.Max/mím & Coste Medio. C. Máx./mím. \\
\hline Director Centro & Media/41.078€ & Media/9.859€ \\
& 64.778 Ceuta & 20.410 Castilla La Mancha \\
& 26.879 C. Valenciana & 9.303 Castilla y León \\
\hline Coordinador Programas & Media/36.073€ & Media/13.133€ \\
& 62.329 Ceuta & 37.412 Castilla y León \\
& 26.161 Madrid & 10.805 C. Valenciana \\
\hline Asesor técnico & Media/32.313€ & Media/12.251€ \\
& 36.525 Canarias & 22.661 Castilla y León \\
& 25.899 Madrid & 2.596 Cataluña \\
\hline Trabajador Social & Media/30.407€ & Media/16.018€ \\
& 55.446 Melilla & 29.115 Andalucía \\
& 22.786 Madrid & 8.404 Extremadura \\
\hline Educador & Media/28.065€ & Media/16.827€ \\
& 53.451 Melilla & 28.352 Canarias \\
& 20.058 Islas Baleares & 5.935 La Rioja \\
\hline Animador/monitor & Media/25.484€ & Media/4.881€ \\
& 32.700 Castilla y León & 12.806 Canarias \\
& 3.781 Islas Baleares & 675 La Rioja \\
\hline Adtivo/Aux.Adtivo & Media/17.790€ & Media/10.742€ \\
& 33.435 Melilla & 83.953 Murcia \\
& 4.322 Aragón & 9.352 C. Valenciana \\
\hline Aux.hogar/Trab. Familiar & Media/16.846€ & Media/9.509€ \\
& 30.869 La Rioja & 21.445 Islas Baleares \\
& 5.821 Castilla-La Mancha & 5.226 Extremadura \\
\hline Servicio Auxiliares/oficios & Media/18.8.02€ & Media/9.874€ \\
& 33.435 Melilla & 12.497 Canarias \\
& 11.407 Islas Baleares & 2.223 Galicia \\
\hline & Media/28.706€ & Media/10.322€ \\
& 55.259 Ceuta & 34.554 Murcia \\
& 11.534 C. Valenciana & 2.034 Castilla La Mancha \\
\hline
\end{tabular}

Memoria Plan Concertado 2005-2006. Elab. Propia.

\section{NO UNA AGENCIA DE VIAJES SINO ACOMPAÑAMIENTO (FUNDAMENTOS Y CLAVES)}

En los apartados anteriores hemos tratado de establecer cómo están las cosas y en qué contexto nos movemos cuando nos referimos a los fundamentos y claves del Trabajo Social y los Servicios Sociales.

A partir de aquí nos interesa repensar aquella apuesta histórica del Trabajo Social por los Servicios Sociales públicos y para todos. En la actualidad, y en el ámbito de los Servicios Sociales, el aumento de la demanda en cantidad y complejidad, así como una creciente oferta y mentalidad de mercado han generado una serie de repercusiones. La primera de ellas es que la unidad de ideas y acción en el Trabajo Social se ha 
resquebrajado. La diversidad de ámbitos de actuación y la lucha por encontrar trabajo propio o relacionado con la profesión ha sido una de las causas. En segundo lugar, los modos de intervenir que los trabajadores han elaborado sobre el campo para hacer frente y para sobrevivir a una nueva organización, ha sido por muchos motivos hostil y contraria a los valores de fondo de la profesión.

De cara al futuro y contando con la organización existente es imprescindible que individualmente se sepa afrontar los problemas planteados contando con la formación recibida y la experiencia acumulada durante años. De este modo es posible que se vayan abriendo formas de trabajar que superen la tensión clásica de los meros enfrentamientos y combinen el trabajo administrativo y el trabajo relacional con los usuarios. Es claramente comprensible que determinadas funciones estrictamente administrativas dejen de ser responsabilidad de los trabajadores sociales; por el contrario es de suma importancia que se acentúe el compromiso contraído con los usuarios de progresar en la solución de lo planteado, se cuide la calidad de las prestaciones y se verifique y haga visible el trabajo realizado mediante la publicación de buenas prácticas. En este último aspecto es necesario el apoyo de la universidad pública, mediante una reconversión de las prácticas y la supervisión profesional de los futuros profesionales.

En base a lo que acabamos de mencionar cobra especial importancia una formación en el management para los trabajadores sociales ${ }^{17}$. También se subraya los puntos de convergencia entre la cultura emprendedora y los objetivos del servicio social, haciendo especial hincapié en el compromiso de los stakholder en la gestión de las respuestas, el énfasis sobre la visibilidad y verificabilidad del trabajo y sobre la calidad de las prestaciones. De este modo se sostiene la importancia de una formación que ponga a los trabajadores a la altura de asumir los desafíos puestos por las nuevas tendencias en los servicios, pero en grado incluso de permanecer fieles a los valores de justicia social propios del servicio social.

En esta misma dirección apuntan las consideraciones de Dal Pra en relación con la formación universitaria que debe tener en cuenta la multifuncionalidad del servicio social ${ }^{18}$. Gorman está en buena medida alineada sobre esta posición: aquello que sirve no obstante según ella es una formación continua, una difusión de la necesidad de aprender despacio el camino y de aprender a conjugar las exigencias emotivas de los usuarios con la nueva organización de los servicios ${ }^{19}$. Roger propone el desarrollo de una búsqueda de cara a la recuperación de los aspectos centrales del rol, perdidos o situados en segundo plano en un contexto en el que la metáfora del mercado y de la empresa privada domina incluso en la organización de los servicios a la persona. Su idea, por tanto más que sobre la formación se centra en su camino de investigación participativa que consientan los trabajadores sociales para conseguir la complejidad del trabajo y del propio recorrido profesional y de vida ${ }^{20}$.

Lo que estamos planteando requiere en el futuro de un mayor número de trabajos empíricos que nos vayan dando luz de por dónde se abren camino las tendencias en materia de Servicios Sociales comunitarios. Ahora que venimos reflexionando sobre el rol de los trabajadores sociales ante el progreso del modelo de estricta o pura gestión que etiquetan otros como care Management, siguen siendo fundamentales cuestiones que hemos venido mencionando: la escucha, el trato humano o cuidado de las relaciones así como los cuidados o participación de las personas. Conviene que los trabajadores sociales lo sigan teniendo claro y arriesguen para encontrarles espacios más amplios donde hacerlos visibles. 
Pensamos que los trabajadores sociales individualmente poco pueden hacer si es que mantenemos la tesis de que los cambios dependen de cada uno de ellos, porque es imposible hacerlo de otro modo. Sin embargo, en ninguno de los últimos congresos realizados en España de trabajadores sociales y de Escuelas de Trabajo Social se han puesto de manifiesto con claridad movimientos claramente contrarios al actual ejercicio del Trabajo Social y los Servicios Sociales. Así las cosas, es conveniente profundizar si la comunidad está de acuerdo o no con el concepto de cuidado que proponen los trabajadores sociales y si éste coincide o no con la idea de care management.

A partir de aquí podríamos considerar dos líneas de actuación que posiblemente conduzcan a un proyecto de ejercicio de la profesión más autónomo. En este sentido es básico el desarrollo de investigaciones específicas de los Servicios Sociales y el Trabajo Social que vayan permitiendo crear y utilizar un patrimonio de conocimientos elaborados en el trabajo diario junto y frente a la diversidad, las fragmentaciones y, desde luego, las conexiones del tejido social.

Si tenemos presente que en los Servicios Sociales comunitarios es la Información y Orientación la prestación más demandada por los mayores $(30,47 \%)$, la familia $(27,11 \%)$, mujer $(7,76 \%)$, discapacitados $(7,14 \%)$ e inmigrantes $(6,27 \%)$, hemos de reflexionar sobre lo que está ocurriendo. Podemos decirlo de modo sencillo subrayando no sólo la incapacidad de los ciudadanos para saber de las cosas de la vida, sino de la falta de otros referentes o fuentes de información y asesoramiento fiables. "Deberíamos decir que muchos hombres son incapaces de construir una narrativa del ego, que les permita reconciliarse con una esfera de la vida personal, cada vez más democratizada y reorganizada" ${ }^{21}$.

Una segunda alternativa de acción estrictamente relacionada con la primera se refiere a la organización de la comunidad profesional y a su capacidad de hacer presión para influir en las políticas sociales. Baste la referencia actual al desarrollo de la Ley de Dependencia en España: existe cierta confusión en los protocolos que se siguen en el conjunto del proceso, así como a la escasez de medios, incluidos los de plantilla, y a la menor o mayor intensidad de prestaciones. Con esta segunda alternativa se trata, fundamentalmente de hacer un frente común con otras profesiones, como aquellas empeñadas en el campo de la educación extra escolástica, de los cuidados de enfermería o con organizaciones de usuarios o de cuidadores.

La idea de que una profesión como el trabajo social pueda ser asimilada a un "nuevo movimiento" es probablemente discutible. Es importante, sin embargo, que se pueda pensar como una comunidad organizada, activa y en grado de orientar las líneas de política social y los modos de ver e interpretar los problemas sobre los que la profesión está llamada a intervenir.

Se puede sostener que la ola de ideología neoliberal a la que está unido el éxito del modelo del care management, con sus connotaciones de individualismo y potenciación de la competitividad únicamente en apariencia presenta puntos de unión con el trabajo social. El problema no es sólo discutir en clave ideológica específicas disposiciones como los buenos servicios, etc., sobre los que, tal vez, se podría incluso poner de acuerdo. Entendemos que lo que expresamos con esta segunda alternativa debe formar parte de los criterios con los que afrontar el futuro de los Servicios Sociales y el Trabajo Social. Debe haber un trabajo de investigación básica y de reflexión crítica y acción por parte de la comunidad/comunidades de profesionales y de sus organizaciones ${ }^{22}$. 
En el cuadro $\mathrm{N}^{\mathrm{o}} 5$ sobre fundamentos y claves del futuro de los Servicios Sociales y el Trabajo Social podemos observar que en la columna de los Fundamentos figuran los contenidos relacionales. Queremos decir que aquellos contenidos relacionales, como los que tienen lugar cuando se establecen los acuerdos entre sujeto de prestaciones de la aplicación de la Ley de Dependencia y el trabajador social para elaborar el PIA (Programa Individual de Atención), son imprescindibles. Ha de añadirse que el usuario tiene la posibilidad de negarse a la propuesta que realiza el trabajador social ${ }^{23}$.Ahora bien, no podemos olvidar que la referencia que acabamos de hacer a la citada Ley de Dependencia, se refiere a establecer las condiciones básicas para poder vivir con cierta dignidad en el caso de las personas dependientes.

Cuadro $\mathrm{N}^{\mathrm{0}}$ 5. Fundamentos y claves del futuro

de los Servicios Sociales y el Trabajo Social

\begin{tabular}{|l|l|}
\hline Fundamentos & Claves \\
\hline Contenidos relacionales & Escuchar a los usuarios \\
\hline Investigación básica & $\begin{array}{l}\text { Asistencia con efectividad tecnológica/Asistencia con relación } \\
\text { humana }\end{array}$ \\
\hline Participación & Rapidez en la gestión \\
\hline Reflexión crítica & Proximidad/Seguimiento \\
\hline Inversión económica & Calidad \\
\hline & Flexibilidad/libertad \\
\hline
\end{tabular}

Como figura en el cuadro $\mathrm{N}^{0} 5$ los contenidos relacionales son importantes, pero es fundamental que se realice una sostenida investigación básica (en los Servicios Sociales Comunitarios) que nos vaya diciendo los cambios que se observan como consecuencia de aplicar determinadas medidas.

La reflexión crítica requiere retomar las cosas para poderlas ajustar de otras posibles formas. Aquella situación que se nos da es lo que nos ofrece el presente; sin embargo para potenciar el futuro se precisa volver a analizar los problemas, las situaciones y hacerlo de modo crítico: no acomodaticio y conservador, sino ofreciendo soluciones con otras posibilidades, con diferentes alternativas, mediante cambios significativos.

Estrechamente relacionadas con los fundamentos de los Servicios Sociales y el Trabajo Social, están las claves que aparecen en el cuadro anterior. Destacaremos la proximidad/seguimiento que sigue siendo algo clave en el conjunto de los Servicios Sociales. Sin la aproximación física, visual y también cultural difícilmente podemos inspirar confianza profesional. El seguimiento es clara expresión del interés y la responsabilidad profesional, o bien que la solución se plantee de modo sostenido, constante, tratando de encauzar el conjunto de variables que intervienen en todo proceso alternativo.

Escuchar a los usuarios es demostrar proximidad y es algo indispensable para poder establecer una serie de contenidos relacionales que puedan dar fruto. Escuchar también supone recabar la información necesaria para poder adaptar a cada caso las soluciones pautadas. Escuchar es ya una forma de mostrar respeto por la persona que decide acudir al profesional a contar, en muchos casos, situaciones que requieren de ayuda y asesoramiento, y que no suelen ser agradables de exteriorizar. Es una de las razones 
elementales para mostrar sensibilidad por los problemas de la persona que escuchamos, equilibrando de algún modo la situación de inferioridad psicológica que porta quien viene como usuario a los Servicios Sociales comunitarios.

Hemos hecho referencia a la Ley de Dependencia en España para ejemplificar el peso específico de contenidos relacionales y otros componentes del proceso que hacen visibles y valorables partes del proceso de cumplimiento de la citada Ley. Sin embargo también hemos señalado que la Ley hace referencia al establecimiento de condiciones básicas para poder vivir con cierta dignidad en el caso de las personas dependientes. No obstante si dejamos el conjunto de las personas dependientes y nos referimos al conjunto de todos los ciudadanos sin distinción, se comprueba que servicios tan elementales como el SAD (Servicio de Ayuda a Domicilio) apenas si superan el 4\% en España. Del mismo modo, el porcentaje de población adulta (15-64 años) que trabaja en los servicios del Estado de Bienestar es del 11,6\% en España, mientras que en Suecia alcanza el 26,9\% siendo la media de la UE-15 el $14,9 \%{ }^{24}$. Con este breve apunte queremos decir que no alcanzamos los mínimos para poder hablar de bienestar en la materia de la que estamos hablando; y por otra parte que, si nos decantamos por la estricta gestión sin apenas subrayar los contenidos relacionales, perdemos también en lo que es más consustancial en el Trabajo Social y los Servicios Sociales comunitarios de calidad.

\section{NOTAS}

${ }^{1}$ Es lo que se plantea en el artículo de Antonio Gutiérrez Resa (2008), "Aplicación de valores en los Servicios Sociales Comunitarios en España”, No de la Rev. Praxis Sociológica (Próxima aparición 2010).

${ }^{2}$ GIDDENS, A., (2006): La transformación de la intimidad. Sexualidad, amor y erotismo en las sociedades modernas, Barcelona Cátedra. ŽIZEK, S. LWMAN, J. y RENDUELES, C. (2008): Arte, Ideología y capitalismo, Madrid, Ediciones Pensamiento, Madrid.

${ }^{3}$ GUTIÉRREZ RESA, A. y MARCUELLO SERVÓS, CH. (2008): Servicios Sociales. Modelos y perspectivas. Una aproximación internacional, Madrid, Ediciones Académicas, pp.72-90.

${ }^{4}$ SLAVOJ Ž. (2007): En defensa de la intolerancia, Madrid, Sequitur, p. 14

${ }^{5}$ RORTY, R. y HABERMAS, J. (2007): Sobre la verdad: ¿Validez universal o justificación?, Madrid, Amorrortu, p. 51

${ }^{6}$ BECK, U. (2002): La sociedad del riesgo global, Madrid, Siglo veintiuno, p. 118.

7 TAYLOR, CH. (1996): Fuentes del yo, Barcelona, Paidós, pp. 38 y 523-524. Consultar en SENNETT, R. (2003): La corrosión del carácter, Barcelona, Anagrama, pp.13-31.

${ }^{8}$ Discurso del profesor Ulrich Beck en el solemne acto académico de investidura como doctor "Honoris Causa" en la UNED de Madrid el 25 de abril de 2007.Consultar BUTLER, J. (2006): Deshacer el género, Barcelona, Paidós Studio 167, pp. 189 y ss.

${ }^{9}$ LACOUR A. y HOEJLUND, H "La sociedad del bienestar en Dinamarca", en GUTIÉRREZ RESA, A. y MARCUELLO SERVÓS, CH. (2008): Servicios Sociales. Modelos y perspectivas. Una aproximación internacional, Madrid, Ediciones Académicas, p. 78.

${ }^{10}$ GUTIÉRREZ RESA, A. (2008): Ontología del mercadillo. Sociología de la vida diaria, Madrid, Ediciones Académicas, p. 182.

11 GUTIÉRREZ RESA, A. y MARCUELlO, CH (2008): Servicios Sociales. Modelos y perspectivas. Una aproximación internacional, Madrid, Ediciones Académicas, p. 77.

${ }^{12}$ GUTIÉRREZ RESA, A. (2007): "Cambio de rumbo en Servicios Sociales y Trabajo Social”, XII Congreso Nacional de Sociología de Castilla-La Mancha, Almagro (Ciudad Real), Asociación Castellano-Manchega de Sociología, p. 49.

${ }^{13}$ ŽIŽEK, S. (2007): En defensa de la intolerancia, Madrid, Sequitur, p. 79.

${ }^{14}$ ŽIŽEK, S. (2007): En defensa de la intolerancia, Madrid, Sequitur, p. 79. 
${ }^{15}$ VV.AA. (2008): La financiación local en España: radiografía del presente y propuestas de futuro, FEMP.

${ }^{16}$ GUTIÉRREZ RESA, A. (2006): Estudio cuantitativo y cualitativo de las familias aragonesas, Zaragoza ,Gobierno de Aragón. Igualmente del mismo autor, (2006): Calidad y futuro de los Servicios Sociales en España (Aragón y Andalucía), Dirección de familia y servicios sociales, Diputación General de Aragón, Zaragoza.

${ }^{17}$ HEALY, K. (2000): Social Work Practiques, London, London Sage.

18 DAL PRA PONTICELLI, M. (2004): Prenderse curae lavoro di cura, Padova, Fundazione Emmanuela Zancan.

19 GORMAN, H. (2000): "Winning hearts and mind? Emotional labour and learning for care manager work", Journal of Social Work Practice, 2.

${ }^{20}$ SPEAR, R. (2005): “L'organizzazione dell'oferta di servizi di welfare en Gran Bretagna”, en Rev. Impresa Sociale, 1.

${ }^{21}$ GIDDENS, A. (2006): La transformación de la intimidad, Madrid, Cátedra, p. 110.

${ }^{22}$ FARGION, S. "Le nuove linee di tendenza nell'organizzazione dei servizi sociali e la professione di assistente sociale", en DAL PARA PONTICELLI, M. (2004): Prendersi cura e lavoro di cura, Padova, Fundazione Emmanuela Zancan, pp. 77-87.

${ }^{23}$ Consejo General de Diplomados en Trabajo Social y Asistentes Sociales (2007), Informe social y programa individual de atención (PIA), Consejo General Colegios Oficiales de Diplomados en Trabajo Social y Asistentes Sociales, Madrid. Importante igualmente es la celebración el día 25 de enero de 2008 del Observatorio Nacional de los Servicios Sociales sobre la implantación de la Ley de Autonomía Personal y Atención a las Personas en situación de Dependencia.

${ }^{24}$ NAVARRO, V. (2007): La situación social en España II, Madrid, Fundación F. Largo Caballero.

\section{BIBLIOGRAFÍA}

BECK, U. (2002): La sociedad del riesgo global, Madrid, Siglo Veintiuno.

- (2007): "Las dialécticas de la modernidad: cómo las crisis de la modernidad surgen de las

conquistas de la modernidad", discurso del profesor Ulrich Beck en el solemne acto académico de

investidura como doctor "Honoris Causa", UNED, Madrid 25 de abril de 2007.

BUTLER, J. (2006): Deshacer el género, Barcelona, Paidós Studio 167.

CONSEJO GENERAL DE DIPLOMADOS EN TRABAJO SOCIAL Y ASISTENTES SOCIALES (2007): Informe social y programa individual de atención (PIA), Consejo General Colegios Oficiales de Diplomados en Trabajo Social y Asistentes Sociales, Madrid.

DAL PRA PONTICELLI, M. (2004): Prendersi cura e lavoro di cura, Padova, Fundazione Emmanuela Zancan.

GIDDENS, A. (2006): La transformación de la intimidad, Madrid, Cátedra.

GORMAN, H. (2000): "Winning hearts and mind? Emotional labour and learning for care manager work", Journal of Social Work Practice, 2.

GUTIÉRREZ RESA, A. (2001): "El Plan Concertado de prestaciones básicas de Servicios Sociales en España (Once años del Plan Concertado)", Rev. Reis n ${ }^{\circ} 93$

- (2006): Calidad y futuro de los Servicios Sociales en España (Aragón y Andalucía), Dirección de familia y servicios sociales. Diputación General de Aragón, Zaragoza.

- (2007): Ontología del mercadillo. Sociología de la vida diaria, Madrid, Ediciones académicas.

- (2007): "Cambio de rumbo en Servicios Sociales y Trabajo Social", XII Congreso Nacional de

Sociología de Castilla-La Mancha, Almagro (Ciudad Real) Asociación Castellano-Manchega de Sociología.

GUTIÉRREZ RESA, A. y MARCUELlO SERVOS, CH. (2008): Servicios Sociales. Modelos y Perspectivas. Una aproximación internacional, Madrid, Ediciones Académicas.

HEALY, K. (2000): Social Work, London Sage.

NAVARRO V. (2207): La situación social en España II, Madrid, Fundación F. Largo Caballero. 
PLAN CONCERTADO (2006): Plan concertado de prestaciones básicas de Servicios Sociales en corporaciones locales, Madrid, Ministerio de Trabajo y Asuntos Sociales.

RORTY, R. y HABERMAS, J. (2007): Sobre la verdad: ¿Validez universal o justificación?, Madrid, Amorrortu.

SENNETT, R. (2003): La corrosión del carácter, Barcelona, Anagrama.

SPEAR, R. (2005): "L'organizzazione dell'oferta di servizi di welfare in Gran Bretagna", en Rev. Impresa Sociale, 1.

TAYLOR, Ch. (1996): Fuentes del yo, Barcelona, Paidós.

VV.AA. (2008): La financiación local en España: radiografía del presente y propuestas de futuro, FEMP. ŽIŽEK, S. (2007): En defensa de la intolerancia, Madrid, Sequitur.

ŽIŽEK, S.; LWMAN, J. y RENDUELES, C. (2008): Arte,_Ideología y capitalismo, Madrid, Ediciones Pensamiento.

\section{Breve currículo:}

\section{Antonio Gutiérrez Resa}

Licenciado en Filosofía Pura en la Universidad de Valencia (1975); Catedrático de Enseñanzas Medias (En excedencia) (1977); -Doctor por la Universidad de Granada (Facultad de Ciencias Políticas y Sociología) (1991); -Catedrático de Servicios Sociales y Trabajo Social en la Universidad de Zaragoza (1996);-Catedrático Servicios Sociales y Trabajo Social en la Universidad Nacional a Distancia (UNED), cuenta con 10 trienios, 6 Quinquenios, 3 Sexenios o tramos de investigación. Últimos libros: Drogodependencias y Trabajo Social, Ediciones Académicas, Madrid, 2007; Servicios Sociales, Modelos y perspectivas. Una aproximación Internacional, Ediciones Académicas, Madrid 2008; Ontología del mercadillo, Ediciones Académicas, Madrid 2007. Artículos: "Sociología de la vida diaria", Rev. Sistema, 2008. "Aplicación de valores en los Servicios Sociales Comunitarios”, Rev. Praxis Sociológica 2009. 